\title{
Effect of mycophenolate mofetil, cyclosporin A, and both in combination in a murine corneal graft rejection model
}

Alexander Reis, Thomas Reinhard, Rainer Sundmacher, Christian Braunstein, Erhard Godehardt

\begin{abstract}
Aims-To compare the effectiveness of mycophenolate mofetil (MMF), cyclosporin A (CSA), and both in combination, in preventing rejection following corneal transplantations.

Methods-Rats of the inbred strains Brown Norway and Lewis were used as donors and recipients respectively. MMF was administered orally in both monotherapy and combination therapy for 14 days in a dosage of $40 \mathrm{mg} / \mathrm{kg}$ body weight, and CSA was administered, likewise for 14 days, in an intramuscular dosage of 10 mg/kg body weight. The transplants were examined every third day by slit lamp microscopy. Every transplant was subjected to histological or immunohistological evaluation.
\end{abstract}

Results-The average transplant survival rate in the allogenic strain combination was 7.9 days (SEM 1.1). Monotherapy with MMF led to a statistically significant prolongation of transplant survival to 11.6 days (SEM 0.9, p< 0.05). Monotherapy with CSA delayed transplant rejection statistically significantly longer than MMF (21 days, 0.0, p< 0.05). The combination therapy with CSA and MMF was statistically significantly superior to the monotherapy with MMF (22.3 days, 0.5, $\mathrm{p}<0.05)$. The combination therapy prolonged transplant survival compared with the CSA monotherapy, albeit not to a statistically significant extent.

Conclusions-In this study we were able to prove the immunosuppressive effect of oral MMF on acute rejection following corneal transplantation. Double drug therapy with CSA and MMF conferred a marginal benefit without a higher incidence of complications related to drug toxicity or overimmunosuppression. (Br F Ophthalmol 1998;82:700-703)

Perforating allogenic corneal transplantation is recognised as a therapy for the treatment of optical, tectonic, and infectious corneal disease. By reason of the positioning of the transplant in an immunologically privileged space (anterior chamber associated immune deviation, $\mathrm{ACAID}^{12}$ ) and the related very good long term results, corneal transplantation has developed into one of the most frequently performed tissue transplantations worldwide. Nevertheless, allogenic rejection represents one of the main reasons for the failure of this form of treatment. There has, it is true, been a clear decline in the incidence of acute rejection after high risk keratoplasties since the introduction of the immunosuppressive antibiotic agent cyclosporin A (CSA). Nevertheless, acute rejection is the cause of over $50 \%$ of transplant opacifications in some immunological high risk groups. ${ }^{34}$ More potent immunomodulating medicaments must be found in order to make extended or individualised therapeutic options for combating rejection available, in particular, to patients with immunological risk factors (for example, stromal vascularisation, retransplantation), and also to patients receiving a particularly immunogenic transplant (limbal keratoplasty ${ }^{5}$ ). One of the most promising immunomodulating substances, whose immunosuppressive effect in combination with CSA following kidney transplantation has already been proved in several clinical studies, is mycophenolate mofetil (MMF, the morpholinoethylester of mycophenolic acid). ${ }^{6-8}$ This substance is an inhibitor of an enzyme which controls purine synthesis at a central point. Mycophenolic acid reversibly inhibits the formation of guanosine nucleotides. ${ }^{9}$ As $\mathrm{T}$ and $\mathrm{B}$ cells are predominantly dependent on a de novo synthesis of guanosine nucleotides, the purine biosynthesis of these cells is selectively inhibited. ${ }^{10}$ Olsen et al performed a dose response study in the rat keratoplasty model and showed the potency of this drug in delaying allograft rejection. ${ }^{11}$

The purpose of this study was to investigate the suitability of the immunosuppressive medicament MMF and the combination CSA/ MMF for suppressing rejection after corneal transplantation, and to compare it with CSA in the prophylaxis of transplant reactions.

\section{Methods}

The corneal transplant was applied to the rat. The strains used in this experiment were Lewis $\left(\mathrm{Rt}^{\mathrm{le}}\right)$ and Brown Norway $\left(\mathrm{Rt}^{\mathrm{ln}}\right)$, (Janvier, France). The animals were obtained and cared for in accordance with the Directives of the European Community as well as with the recommendations of the NIH Guide for the Care and Use of Laboratory Animals, National Institutes of Health Publication No 85-23 (revised 1985).

OPERATIVE TECHNIQUE

We conducted an orthotopic perforating keratoplasty according to the technique of Herbort et al. ${ }^{12}$ A medicamentous mydriasis in
Accepted for publication 21 January 1998 
the eyes of both the donors and the recipients was achieved by the local application of phenylephrine $10 \%$ eye drops. These eye drops were administered three times at intervals of 10 minutes before the operation. During an inhalation anaesthesia with diethyl ether the corneas of the donor rats (Brown Norway, BN) were obtained by the use of a $3.5 \mathrm{~mm}$ trepan. Until their implantation, the donor buttons were stored at room temperature for approximately 20 minutes in a conservation medium for corneas (Likorol, Opsias, France).

The recipient animals (Lewis) were pretreated in the same way and, after a brief inhalation anaesthesia with diethyl ether, were anaesthetised with an intraperitoneal mixed injection of ketamine hydrochloride $100 \mathrm{mg} / \mathrm{kg}$ body weight, midazolam $0.5 \mathrm{mg} / \mathrm{kg}$ body weight, and atropine $0.5 \mathrm{mg} / \mathrm{kg}$ body weight, and fixed in a dextral lateral position. After the left host cornea had been removed with a 3.0 $\mathrm{mm}$ trepan, the donor cornea was transplanted. The transplant was sewn in with eight interrupted sutures (Ethicon 11.0). The anterior eye chamber was restored at the end of the operation by the instillation of balanced salt solution. To protect the transplant, a blepharorrhaphy was attached by means of two interrupted sutures (Prolene 6.0) and remained in place for 3 days, and Refobacin (gentamicin) was applied in the palpebral fissure.

The groups were divided up as follows: group 1 (six animals): Lewis/Lewis (this control was conducted in order to determine the influence of the operation on the cornea in the absence of transplantation immunological phenomena, control 1)

group 2 (eight animals): BN/Lewis (no therapy, control 2)

group 3 (seven animals): BN/Lewis (CSA, 10 $\mathrm{mg} / \mathrm{kg}$ body weight/day, intramuscularly)

group 4 (seven animals): BN/Lewis (MMF, 40 $\mathrm{mg} / \mathrm{kg}$ body weight/day, oral; this dosage was chosen according to the dose response curves done by Olsen et al ${ }^{12}$ )

group 5 (seven animals): BN/Lewis (cyclosporin $\mathrm{A}, 10 \mathrm{mg} / \mathrm{kg}$ body weight/day intramuscularly and MMF $40 \mathrm{mg} / \mathrm{kg}$ body weight/day, oral).

Medication in the therapy groups was started on the day of operation and continued daily for 14 days.

CLINICAL EVALUATION

All rats were subjected to a clinical examination twice a week for at most 8 weeks. Each animal was examined by slit lamp microscopy during a brief inhalation anaesthesia with diethyl ether. The transplants were evaluated by means of a scoring system which assessed opacity, oedema, and neovascularisation.

Opacity: 0 = no opacity; 1 = slight opacitydetails of iris clearly visible; $2=$ some details of iris no longer visible; $3=$ pronounced opacity-pupil still recognisable; $4=$ total opacity.

Oedema: $0=$ no oedema; 1 = mild oedema; $2=$ pronounced oedema with raised transplant.
Neovascularisation: $0=$ no vessels; $1=$ vessels in the periphery; $2=$ vessels extending deeper; 3 = vessels extending to the centre.

The target criterion was complete opacification (rejection) of the transplant. As soon as the donor cornea had been clinically identified as rejected the animal was sacrificed through the inhalation of carbon dioxide.

HISTOLOGICAL AND IMMUNOHISTOLOGICAL EVALUATION

After 8 weeks, or following clinically diagnosed complete opacification of the transplant, the recipient animals were killed through the inhalation of carbon dioxide. After that, the operated eye was enucleated and fixed in a buffered formalin solution (4\%). For histological assessment the formalin fixed eyes were cut into $4 \mu \mathrm{m}$ thick preparations and subjected to haematoxylin and eosin or elastica staining. For the immunohistological evaluation the 4 $\mu \mathrm{m}$ thick preparations were pretreated with a Histosafe Enhancer (Serotec, Canada) to improve antibody affinity. Following this, the preparations were subjected to an immunohistological examination using the avidin-biotinperoxidase complex method. The primary monoclonal antibody used was MCA 48g (Serotec, Canada), which reacts with rat CD8 antigen. The secondary antibody, a biotin marked rabbit-antimouse antibody, and the reagents for the third phase reaction, were likewise obtained from the Serotec company (Vectastain Elite ABC Peroxidase Kit). As the substrate for the peroxidase, 3-3 diaminobenzidine (Peroxidase Substrate Kit) was used.

\section{STATISTICAL ANALYSIS}

The time from transplantation to the opacification of the transplant was compared between the various groups by means of the MannWhitney U test.

\section{Results}

\section{CLINICAL EVALUATION}

Figure 1 shows the transplant survival rate in the control and therapy groups. Transplants which opacified in the first 3 days after operation were omitted from the statistics (two animals in group 2, one animal in group 3) as representing errors of operative technique (bleeding, suture dehiscence, lens opacification). Cornea transplantation in the syngeneic combination (group 1, Lewis/Lewis) led to slight perioperative stromal transplant oedema which was no longer detectable from the sixth postoperative day onwards. Throughout the entire period of the examination the transplants remained clear. Neovascularisation was detectable only in the area of the sutures. The average transplant survival rate in the allogenic combination (group 2, BN/Lewis) was 7.9 days (SEM 1.1). The monotherapy with MMF (group 4) led to a statistically significant prolongation of transplant survival to 11.6 days (SEM 0.9, p<0.05). The monotherapy with CSA (group 3) delayed transplant rejection statistically significantly longer than MMF (21 days, $0.0, \mathrm{p}<0.05)$. The combination therapy with CSA and MMF (group 5) was superior to 


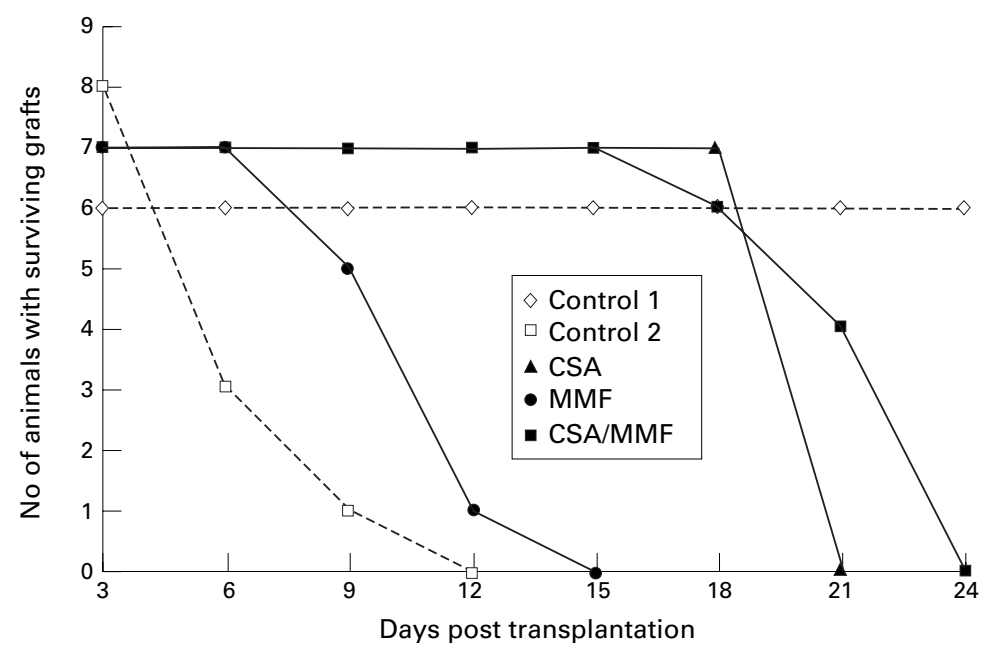

Figure 1 Transplant survival rate in the control and therapy groups.

a statistically significant extent to the monotherapy with $\mathrm{MMF}$ (22.3 days, $=0.5, \mathrm{p}<0.05)$. The combination therapy prolonged transplant survival compared with CSA monotherapy, albeit not to a statistically significant extent.

HISTOLOGY AND IMMUNOHISTOLOGY

In the eighth postoperative week the transplants of the syngeneic control group (group 1 ), showed, only in the area of the interrupted sutures, isolated foreign body giant cells and a low level of mononuclear infiltrate. The central periphery and the centre of the transplant revealed no cellular infiltration whatsoever, and the configurations of epithelium, stroma, and endothelium were normal. The allotransplants of group 2 showed, after rejection, a pronounced oedema characterised by an extreme thickening of the stroma and the development of vacuoles, particularly in the area of the basal membrane of the epithelium, with the histological picture of a bullous keratopathy. A pronounced mononuclear infiltration was to be found in all layers of the transplants, but was most pronounced in the area of the stroma and the deeper layers of the epithelium. An extremely dense mononuclear infiltrate was detectable at the edge of the transplant in the area of the interrupted suture, where an accumulation of macrophages was also to be found.

Immunohistological staining with MCA $48 \mathrm{~g}$, a monoclonal antibody, which reacts with rat CD8 antigen revealed that both in the MMF and the CSA treated transplants approximately one third of the infiltrating lymphocytes were $\mathrm{CD} 8+$ at the time of complete rejection. There were no significant differences in the histomorphological and immunohistological picture of the MMF and the CSA treated grafts.

\section{COMPATIBILITY OF MMF}

The rats of the therapy groups showed no increase in weight during the first 14 days after operation. Altogether, three of 14 rats which received MMF (groups 4 and 5) showed diarrhoea in the first postoperative week. However, with no reduction in dosage, this clinical symp- tom was no longer observable in the second postoperative week.

\section{Discussion}

Some patients considered at high risk of corneal graft rejection are given systemic cyclosporin A. This prophylaxis is not sufficient or compatible after all high risk keratoplasties. The purpose of this study was to compare the efficacy of CSA, MMF, and both in combination, in preventing rejection following corneal transplantations.

In this study we have been able to prove the immunosuppressive effect of $\mathrm{MMF}$ in delaying rejection after allogenic corneal transplantation. The monotherapy with MMF produced a statistically significantly prolonged transplant survival $(\mathrm{p}<0.05)$ compared with the allogenic control group. However, the monotherapy with CSA was statistically significantly superior to the monotherapy with MMF $(\mathrm{p}<0.05)$. The combination therapy with CSA and MMF led to the longest postoperative transplant survival, but statistical significance was achieved only in comparison with the MMF monotherapy.

The histological examination was performed on clinically rejected transplants, and showed, almost monomorphously, a massive cellular infiltration of the transplant by mononuclear cells. Antigen presenting cells (monocytes/ macrophages) were found, particularly in the area of the interrupted sutures, where a local accumulation of lymphocytes spreading from the recipient cornea was also evident.

The immunohistological evaluation was carried out after rejection had taken place and revealed that both in the MMF and CSA treated grafts approximately one third of the infiltrating lymphocytes were CD8+. This is consistent with previous findings in this model of corneal transplantation. ${ }^{13} \mathrm{MMF}$ and CSA did not cause a different pattern of cellular infiltration. A statistically significant difference between these two therapeutic regimens was only found in the protraction of the rejection process.

In this study it was again possible to observe the enterotoxicity of MMF as already described in previous studies. ${ }^{6-8}$ This can be explained partially by the enterohepatic circulation of MMF. However, the rats treated with MMF recovered even while it was still being administered, and with no reduction in dosage.

A large number of new immunosuppressive drugs are currently undergoing clinical testing. To date, only one of those not derived from CSA has been clinically proved to possess superior efficacy to CSA - that is, FK-506. ${ }^{14}$ MMF is distinguished by a synergistic effect in relation to CSA combined with a low rate of side effects and considerable therapeutic breadth. This medicament makes possible immunosuppression oriented even more specifically to individually differing risk constellations.

In this study we were able to prove the immunosuppressive effect of oral $\mathrm{MMF}$ on acute rejection following corneal transplantation. Double drug therapy with CSA and 
MMF conferred a marginal benefit without a higher incidence of complications related to drug toxicity or overimmunosuppression.

1 Niederkorn JY, Mellon J. Anterior chamber-associated immune deviation promotes corneal allograft survival. Invest Ophthalmol Vis Sci 1996;37:2700-7.

2 Streilen JW. Peripheral tolerance induction: lessons from immune privileged sites and tissues. Transplant Proc 1996;28:2066-70.

3 Reinhard T, Sundmacher R, Heering P. Systemic cyclosporin A in high risk keratoplasties. Graefes Arch Clin Exp Ophthalmol 1996;234:115-121

4 Sundmacher R, Reinhard T, Heering P. Six years experience with systemic ciclosporin A prophylaxis in high-risk perforatwith systemic ciclosporin A prophylaxis in high-risk perforat-
ing keratoplasty patients. Ger $\mathcal{F}$ Ophthalmol 1992;1:432-6.

5 Sundmacher R, Reinhard T. Central corneolimbal transplantation under systemic ciclosporin A cover for severe limbal stem cell insufficiency. Graefes Arch Clin Exp Ophthalmol 1996;234:122-5.

6 Deierhoi M, Sollinger H, Diethelm A, et al. One-year follow-up results of a phase 1 trial of mycophenolate mofetil (RS-61443) in cadaveric renal transplantation. Transplant Proc 1993;25:693.

7 European Mycophenolate Mofetil Cooperative Study Group. Placebo controlled study of mycophenolate mofetil combined with cyclosporin and corticosteroids for the prevention of acute rejection. Lancet 1995;345:1321-5.
8 The Tricontinental Mycophenolate Mofetil Renal Transplantation Study Group. A blinded, randomized clinical trial of mycophnolate mofetil for the prevention of acute rejection in cadaveric renal transplantation. Transplantation 1995;61:1029-37.

9 Allison A, Hovi R, Watts A, et al. The role of de novo purine synthesis in lymphocyte transformation. Ciba Found Symp 1977;48:207.

10 Morris R, Hoyt E, Murphy P. Mycophenolic acid morpholinoethylester (RS-61443) is a new immunosuppressant that prevents and halts heart allograft rejection by selevtive inhibition of T- and B-cell purine synthesis. Transplant Proc 1990;22:1659.

11 Olsen TW, Joplin A, Anderson EE, et al. RS-61443 prevents corneal allograft rejection in the orthotopic rat penetrating keratoplasty model. Annual Meeting of the Investigative Ophthalmology and Visual Science, Sarasota, Florida, USA; 1-6 May 1994. Invest Ophthalmol Vis Sci 1994;35: 1877 .

12 Herbort CP, Matsubara M, Mishi M, et al. Penetrating keratoplasty in the rat: a model for the study of immunosuppressive treatment of graft rejection. $\mathcal{F}_{p n} \mathcal{F}$ Ophthalmol 1989;33:212-20.

13 Hikita N, Lopez JS, Chan C, et al. Use of topical FK506 in a corneal graft rejection model in lewis rats. Invest Ophthalmol Vis Sci 1997;38:901-9.

14 Hooks $M$. Tacrolimus, a new immunosuppressant-a review of the literature. Ann Pharmacother 1994;28:501. 